\title{
Surface Display of AcMNPV Occlusion-Derived P74 Does Not Enhance Oral Infectivity of Budded Viruses
}

\author{
Victoria Alfonso María Gabriela López Elisa Carrillo Oscar Taboga
}

Instituto de Biotecnología, CICVyA, INTA-Castelar, Hurlingham, Argentina

\section{Key Words}

Baculovirus $\cdot$ P74 $\cdot$ Pseudotyping $\cdot$ Budded virus •

Autographa californica multiple nucleopolyhedrovirus

\begin{abstract}
Baculovirus occlusion-derived viruses (ODVs) and budded viruses (BVs) are morphologically and functionally distinct. ODVs are responsible for primary infection in insect hosts because of their high per os infectivity. On the contrary, BVs poorly infect endothelial gut cells, but propagate the infection in the tissues of insects with a high efficiency. P74 is one of the most important proteins from ODVs, and it participates in the attachment of this viral phenotype to endothelial cells in the midgut. We evaluated the possibility of pseudotyping BVs of Autographa californica multiple nucleopolyhedrovirus with two versions of P74 and its effect on their oral infectivity. Both recombinant BVs contained P74 and replicated similarly to wild-type viruses. Nevertheless, the presence of P74 on the BV's surface does not enhance the oral infectivity of this phenotype, suggesting that the presence of P74 in the membrane of budded virions interferes with their mechanism of infecting midgut cells.
\end{abstract}

Copyright $\odot 2011$ S. Karger AG, Basel

\section{KARGER}

Fax +41613061234 E-Mail karger@karger.ch www.karger.com
(C) 2011 S. Karger AG, Basel

$0300-5526 / 12 / 0553-0247 \$ 38.00 / 0$

Accessible online at:

www.karger.com/int
Baculoviruses belong to a broad family of virus pathogens of insects. Two distinct infectious phenotypes exist in their life cycle: occlusion-derived viruses (ODVs) and budded viruses (BVs) [1]. ODVs are embedded within a polyhedrin matrix, forming the baculovirus occlusion bodies or polyhedra. When larvae feed on contaminated leaves, polyhedra dissolve in the midgut and release ODVs which infect the epithelial cells initiating primary infection [2]. The uptake of ODVs is mediated by specific factors of attachment and fusion to cell membranes, although the mechanisms involved in this process remain poorly understood.

In Autographa californica multiple nucleopolyhedrovirus (AcMNPV), the most deeply studied member of the Baculoviridae family, it is known that some ODV proteins, like P74 and other per os infectivity factors or PIFs, are essential to infect larvae by the oral route [3-5]. The p74 gene is expressed at the late stage of infection [4] and encodes a 645-amino acid protein that is located exclusively on the ODV lipid bilayer envelope and is exposed on the ODV surface [6].

Yao et al. [7] and Zhou et al. [8] showed that a soluble form of P74 is capable of rescuing the infectivity of P74null ODVs, indicating that this protein has an essential role in viral adsorption or fusion with the target cell. Nevertheless, how the P74 protein interacts with host midgut proteins still remains unknown. BVs bud from the baso- 
lateral side of the infected midgut cells and infect tissues in the hemocoel. A baculovirus envelope glycoprotein called GP64 is involved in BV cell attachment and is required for adsorptive endocytosis followed by the fusion of the viral and endosomal membranes [9]. It has been reported that BVs have a very low infectivity in endothelial cells of the larval midgut [10]. Keddie and Volkman [11] showed that BVs are $10^{6}$-fold less infectious in the midgut than in the hemocoel; for comparison, ODVs are $10^{4}$-fold more orally infectious than BVs.

Pseudotyping is a successful strategy, used mainly in gene therapy, and consists of modifying viral tropism through the surface display of foreign proteins or peptides that interact with receptors present in certain cell types [12]. The display of antigens or other proteins on budded baculovirus envelopes, mainly fused with GP64, has been extensively studied and has become a powerful tool for modifying viral tropism, displaying antigens or studying the role of heterologous membrane proteins in the baculovirus context [13-19].

In many laboratories, the baculovirus-insect cell system is broadly used to express antigens of medical and veterinary interest. Recombinant viruses in which genes of interest are introduced into the polyhedrin locus do not produce occlusion bodies (occ-). We hypothesized that BV oral infectivity could be improved by bringing budded virions displaying P74 closer to the midgut cells, taking advantage of the interaction of P74 with its cellular receptor. This approach would contribute to the development of a method for the production of antigens expressed by occ- recombinant baculoviruses delivered by the oral route.

With the purpose of displaying $\mathrm{P} 74$ protein on $\mathrm{BV}$, two recombinant baculoviruses were designed: AcP74exSup and $A c$ P74Sig. In these constructs, the strong very late polyhedrin promoter drove the expression of a second copy of P74 inserted into the polyhedrin locus. In order to expose a portion of P74 in the BV's peplomers, $A c \mathrm{P} 74$ exSup virus was constructed by fusing a truncated form of P74 (P74ex) externally located in wild-type (wt) ODVs (AcMNPV complete genome NC 001623, nucleotides 119,794 to 121,069$)$ with a second copy of the viral glycoprotein GP64. The other strategy consisted of distributing P74 on the viral envelope, anchoring it to the cell membrane by its own transmembrane domain, taking advantage of baculoviruses passively acquiring P74 during the budding process. To obtain AcP74Sig, the complete sequence of p74 omitting the start codon (AcMNPV nucleotides 119,135 to 121,069) was fused with the gp64 signal peptide. Recombinant AcP74exSup and
AcP74Sig were obtained using BaculoGold ${ }^{\mathrm{TM}}$ methodology (Pharmingen). Immunoblotting of cell lysates demonstrated the expression of P74 in cells infected with both recombinant baculovirus, and time course analysis of $\mathrm{BV}$ production showed similar virus yields to wt AcMNPV (data not shown).

The exposure of both recombinant forms of P74 on the surface of cell membranes was evidenced by immunofluorescence assays (fig. 1a). Upon infection with either recombinant baculovirus, AcP74Sig or AcP74exSup, nonpermeabilized cells displayed positive staining for the recombinant P74s on cell surfaces with a specific antibody against P74 (N25 8c [6]) and a FITC-conjugated goat anti-mouse antibody (BD Biosciences). In contrast, fluorescence staining was absent on cells infected with wt AcMNPV. A control with anti-GP64 (AcV5 [20]) was included to show that viral proteins can be detected at the cell surface. This analysis suggests not only the presence, but also the display, of P74 in BV membranes. Consequently, preparations of each purified BV [21] were examined by immunoblotting to determine the association of P74 to the virions.

As shown in figure $1 \mathrm{~b}$, an antibody against P74 revealed bands of 110 and $74 \mathrm{kDa}$, according to the case of P74 fused to GP64 or P74, respectively. P74 was not detected in wt AcMNPV BVs. Western blot analysis using an antibody against GP64 revealed the $110-\mathrm{kDa}$ GP64P74 fusion product in AcP74exSup and wt GP64 in wt AcMNPV BVs and in AcP74exSup. Therefore, as expected, P74 was readily detected in BVs purified from cells infected with recombinant baculoviruses, although at least $200 \mathrm{ml}$ of culture supernatants $\left(5 \times 10^{7}\right.$ plaqueforming units, $\mathrm{pfu} / \mathrm{ml}$ ) had to be processed to visualize immunoblotting bands, suggesting that P74 was poorly represented in the virions. Likewise, wt p74 is a late gene weakly transcribed [4], and the low quantity of P74 has made it difficult to investigate. Besides, although P74 plays a crucial role in the oral route of infection, the overexpression of $\mathrm{P} 74$ protein does not enhance the pathogenicity of viral occlusion bodies [8].

It is known that viral attachment and fusion of membranes are processes that need the participation of more than one protein. At least PIFs 1, 2 and 3 and P74 are all required for ODV oral infection. Slack et al. [22] have shown that a truncated mutant of P74 lacking its transmembrane anchor is able to keep its functionality, and it has been suggested that P74 could form a complex with other envelope proteins. Recently, Peng et al. [23] have demonstrated that PIFs form a complex and that P74 is associated with it. Although budded baculoviruses lack 
Fig. 1. a Detection of recombinant P74 by immunofluorescence in infected cells. Sf-9 cells were infected with AcP74exSup, AcP74Sig or wt AcMNPV at a multiplicity of infection of $10 \mathrm{pfu}$. At 2 days postinfection, cells were probed with anti-P74 antibody or anti-GP64 antibody and visualized using FITC-conjugated goat antimouse IgG. b Analysis of recombinant P74 in purified budded virions. AcP74exSup BVs, AcP74Sig BVs and wt AcMNPV BVs were purified by a sucrose gradient and analyzed by SDS-PAGE and Western blot. Blots were probed with anti-P74 and antiGP64 antibodies.



PIFs, it has been shown that they are able to initiate an infection by the oral route in the larval hosts with low efficiency. Thus, P74-pseudotyping could improve the binding of BVs to insect gut cells since the interaction of P74 to brush border membrane vesicles has previously been proven [24].

With the purpose of determining whether displaying recombinant P74 or P74ex on BVs was able to induce changes in AcMNPV oral infectivity, virus yields were measured in a bioassay of oral infection of Rachiplusia $n u$ and compared against nonrecombinant BV infectivity. Bioassay was not carried out by scoring mortality because most larvae do not die from BV feeding. Groups of 27 third instar $R . n u$ larvae fed individually with a diet containing $6 \times 10^{6}$ pfu of AcP74exSup BV, AcP74Sig BV, wt AcMNPV BV or PBS were daily monitored.

Twenty-four hours or 4 days postinfection viral DNA was extracted from hemolymph and quantified by qPCR using SYBR Green PCR Master Mix (Applied BioSystems) and the qPCR primers VP39forN 5' cgacaaatgaga- gttaatcgctgc $3^{\prime}$ and VP39RCuan 5' gtcgtcttcgtcgaaaatgggcaa 3', which were designed to amplify a 183-bp genomic fragment of the vp39 gene. Samples were analyzed in a 7500 Real Time PCR System using the 7500 System Software (Applied BioSystems). Viral DNA was not detected in either experimental group at $24 \mathrm{~h}$ postinfection. However, as depicted in figure 2, viral DNA was detected in samples taken at the 4 th day postinfection and a high dispersion was noticed in QPCR values among individual larvae from each experimental group. Analysis of the data revealed, on the contrary to what we expected, that recombinant viruses were not more infectious than wt.

The presence of P74 in BVs was associated with a high number of samples with values of viral DNA in hemolymph below quantification limit $(\mathrm{p}=0.0041 \mathrm{AcP} 74 \mathrm{Sig}$ vs. wt $A c \mathrm{MNPV}, \mathrm{p}=0.0108$ AcP74exSup vs. wt $A c \mathrm{MNPV}$; Fisher's exact test). As expected, larvae fed with a PBScontaminated diet reached DNA values below the quantification limit. Similar results were obtained when viral titers in hemolymph were determined by plaque assays 
Fig. 2. Quantitative real-time PCR analysis of viral DNA. $R$. nu larvae were fed with a diet containing PBS or $6 \times 10^{6} \mathrm{pfu}$ of AcP74exSup BV, AcP74Sig BV or wt AcMNPV BV. At 4 days postinfection, DNA was extracted from hemolymph and analyzed by qPCR. The dotted line represents the assay's quantification limit, calculated as the lowest DNA concentration in the standard curve multiplied by the dilution factor.

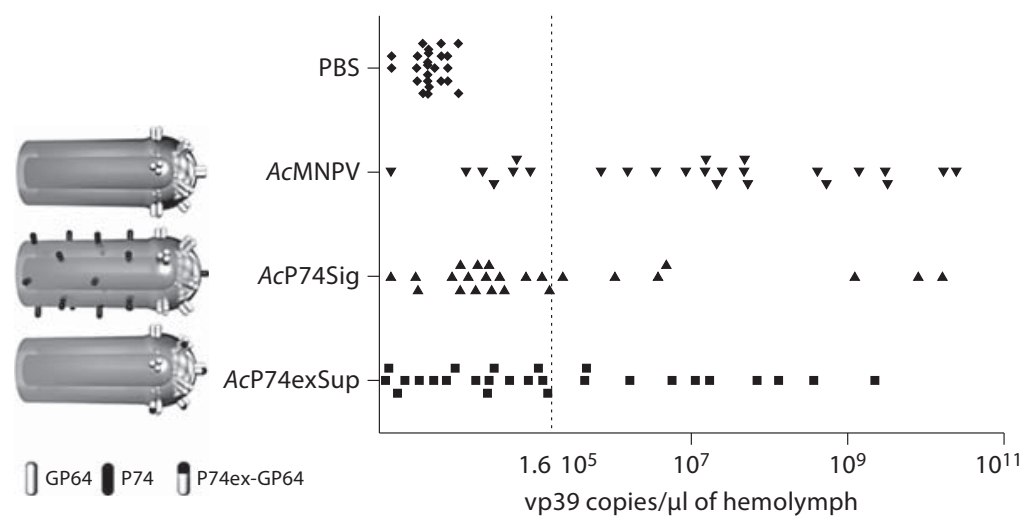

(data not shown). These results demonstrated that the presence of P74 in BVs does not improve the oral infectivity in $R . n u$ larvae, suggesting that a major proximity of the virus to the target cells is not the only condition to increase the natural infectivity of this viral phenotype and that P74 by itself is not capable of modifying BV tropism. However, we cannot rule out the incorrect folding of P74 in both recombinants, and the spatial conformation of P74 present in BV may not be adequate for achieving a biological role.

Moreover, a diminished infectivity was observed in recombinant BVs compared with wt BVs. Since growth curves performed in cultured cells with AcP74exSup BV, AcP74Sig BV and wt viruses revealed that $\mathrm{P} 74$ protein present on the surface of BV does not interfere with BV entry into cells, modified viruses retain the ability to infect cells in a secondary infection on the same level as wt. Nevertheless, it is possible that P74 modifies virus entry to midgut cells, altering the interaction between an unknown cell receptor and the virus ligand. On the other hand, this steric impediment would be also capable of disturbing ODV attachment to endothelial cells. Perhaps BVs are not able to infect cells of the midgut, and the per os infection of larvae with wt BVs is due to the presence of a pre-occluded virus or POVs in supernatants of infected cells as a product of lysis that co-purify in small quantities with BVs. Therefore, a plausible explanation could be that the decrease in infectivity observed for $A c$ P74exSup and AcP74Sig BVs is due to the interference of pseudotyped BVs to contaminating POVs.

In this paper, our results demonstrate that the surface display of P74 protein on budded baculoviruses is not an adequate tool to raise their per os infectivity, and it is possible that it interferes with the mechanism used by this phenotype to infect midgut cells. Further investigation is required to understand in more detail the different pathways used by the two baculoviral phenotypes to orally infect lepidopteran larvae.

\section{Acknowledgements}

This work was supported by grants PICT 2007716 ANPCyT and PE INTA 2009-2012 AERG232161. Dr. Victoria Alfonso, Dr. Elisa Carrillo and Dr. Oscar Taboga are members of the CONICET Research Carrier Programme (Consejo Nacional de Investigaciones Científicas y Técnicas). Larvae were kindly provided by Ing. Agr. Mariela Fogar from INTA Saenz Peña, Chaco, Argentina, and maintained by Mrs. María Cornacchio and Mrs. Débora Moreyra from IMYZA, INTA Castelar. The authors thank Dr. Gary Blissard of Boyce Thompson Institute for Plant Research at Cornell University, Ithaca, N.Y., USA, for providing anti-P74 mAb N25 8c.

\section{References}

1 Williams GV, Faulkner P: Cytological changes and viral morphogenesis during baculovirus infection; in Miller LK (ed): The Baculoviruses. New York, Plenum Press, 1997, pp 61-107.

2 Granados RR, Lawler KA: In vivo pathway of Autographa californica baculovirus invasion and infection. Virology 1981;108:297-302.

- 3 Kikhno I, Gutiérrez S, Croizier L, Croizier G, Ferber ML: Characterization of pif, a gene required for the per os infectivity of Spodoptera littoralis nucleopolyhedrovirus. J Gen Virol 2002;83:3013-3022. 
-4 Kuzio J, Jaques R, Faulkner P: Identification of p74, a gene essential for virulence of baculovirus occlusion bodies. Virology 1989;173: 759-763.

5 Pijlman GP, Pruijssers AJ, Vlak JM: Identification of pif-2, a third conserved baculovirus gene required for per os infection of insects. J Gen Virol 2003;84:2041-2049.

-6 Faulkner P, Kuzio J, Williams GV, Wilson JA: Analysis of p74, a PDV envelope protein of Autographa californica nucleopolyhedrovirus required for occlusion body infectivity in vivo. J Virol 1997;78:3091-3100.

7 Yao L, Zhou W, Xu H, Zheng Y, Qi Y: The Heliothis armigera single nucleocapsid nucleopolyhedrovirus envelope protein P74 is required for infection of the host midgut. Virus Res 2004;104:111-121.

$\checkmark 8$ Zhou W, Yao L, Xu H, Yan F, Qi Y: The function of envelope protein P74 from Autographa californica multiple nucleopolyhedrovirus in primary infection to host. Virus Genes 2005;30:139-150.

9 Volkman LE, Goldsmith PA: Mechanism of neutralization of budded Autographa californica nuclear polyhedrosis virus by a monoclonal antibody: iInhibition of entry by adsorptive endocytosis. Virology $1985 ; 143$ : 185-195.

10 Cha HJ, Dalal NG, Pham M-Q, Vakharia VN, Rao G, Bentley WE: Insect larval expression process is optimized by generating fusions with green fluorescent protein. Biotechnol Bioeng 1999;65:316-324.
$>11$ Keddie BA, Volkman LE: Infectivity difference between the two phenotypes of Autographa californica nuclear polyhedrosis virus: importance of the $64 \mathrm{~K}$ envelope glycoprotein. J Gen Virol 1985;66:1195-1200.

12 Mäkelä AR, Oker-Blom C: Baculovirus display: a multifunctional technology for gene delivery and eukaryotic library development. Adv Virus Res 2006;68:91-112.

13 Tami C, Farber M, Palma E, Taboga O: Presentation of antigenic sites from foot andmouth disease virus on the surface of baculovirus and in the membrane of infected cells. Arch Virol 2000;145:1815-1828.

14 Oker-Blom C, Airenne KJ, Grabherr R: Baculovirus display strategies: emerging tools for eukaryotic libraries and gene delivery. Brief Funct Genomic Proteomic 2003;2:244253.

15 Tani H, Limn CK, Yap CC, Onishi M, Nozaki M, Nishimune Y, Okahashi N, Kitagawa Y, Watanabe R, Mochizuki R, Moriishi K, Matsuura $\mathrm{Y}$ : In vitro and in vivo gene delivery by recombinant baculoviruses. J Virol 2003;77:9799-9808.

16 Facciabene A, Aurisicchio L, La Monica N: Baculovirus vectors elicit antigen specific immune responses in mice. J Virol 2004;78: 8663-8672.

17 Tami C, Peralta A, Barbieri R, Berinstein A Carrillo E, Taboga O: Immunological properties of FMDV-gp64 fusion proteins expressed on Sf9 cell and baculovirus surfaces. Vaccine 2004;23:840-845.
18 Kitagawa Y, Tani H, Limn CK, Matsunaga TM, Moriishi K, Matsuura Y: Ligand-directed gene targeting to mammalian cells by pseudotype baculoviruses. J Virol 2005;79: 3639-3652.

19 Ernst W, Schinko T, Spenger A, Oker-Blom C, Grabherr R: Improving baculovirus transduction of mammalian cells by surface display of a RGD-motif. J Biotechnol 2006; 126:237-288.

20 Hohmann AW, Faulkner P: Monoclonal antibodies to baculovirus structural proteins: determination of specificities by Western blot analysis. Virology 1983;125:432-444.

21 O’Reilly DR, Miller LK, Luckow VA: Baculovirus Expression Vectors: A Laboratory Manual. New York, Freeman WH and Co., 1994.

-22 Slack JM, Lawrence SD, Krell PJ, Arif BM: A soluble form of P74 can act as a per os infectivity factor to the Autographa californica multiple nucleopolyhedrovirus. J Gen Virol 2010;91:915-918.

-23 Peng K, van Oers MM, Hu Z, van Lent JWM, Vlak JM: Baculovirus per os infectivity factors form a complex on the surface of occlusion-derived virus. J Virol 2010;84:94979504.

-24 Haas-Stapleton EJ, Washburn JO, Volkman LE: P74 mediates specific binding of Autographa californica $\mathrm{M}$ nucleopolyhedrovirus occlusion-derived virus to primary cellular targets in the midgut epithelia of Heliothis virescens larvae. J Virol 2004;78:6786-6791. 